\title{
The Chx10-Traf3 Knockout Mouse as a Viable Model to Study Neuronal Immune Regulation
}

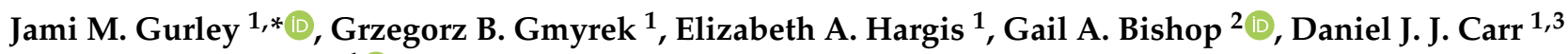 \\ and Michael H. Elliott ${ }^{1}$ (D)
}

1 Department of Ophthalmology, Dean McGee Eye Institute, University of Oklahoma Health Sciences Center (OUHSC), 608 Stanton L. Young Blvd., Oklahoma City, OK 73104, USA; grzegorz-gmyrek@ouhsc.edu (G.B.G.); elizabeth-hargis@ouhsc.edu (E.A.H.); dan-carr@ouhsc.edu (D.J.J.C.); michael-elliott@ouhsc.edu (M.H.E.)

2 Department of Microbiology and Immunology, University of Iowa and VAMC, Iowa City, IA 52242, USA; gail-bishop@uiowa.edu

3 Department of Microbiology and Immunology, University of Oklahoma Health Sciences Center (OUHSC), 608 Stanton L. Young Blvd., Oklahoma City, OK 73104, USA

* Correspondence: jami-gurley@ouhsc.edu

check for updates

Citation: Gurley, J.M.; Gmyrek, G.B.; Hargis, E.A.; Bishop, G.A.; Carr, D.J.J.; Elliott, M.H. The Chx10-Traf3 Knockout Mouse as a Viable Model to Study Neuronal Immune Regulation. Cells 2021, 10, 2068. https://doi.org/ 10.3390/cells10082068

Academic Editor: Paola Bagnoli

Received: 2 July 2021

Accepted: 9 August 2021

Published: 12 August 2021

Publisher's Note: MDPI stays neutral with regard to jurisdictional claims in published maps and institutional affiliations.

Copyright: (c) 2021 by the authors. Licensee MDPI, Basel, Switzerland. This article is an open access article distributed under the terms and conditions of the Creative Commons Attribution (CC BY) license (https:// creativecommons.org/licenses/by/ $4.0 /)$.
Abstract: Uncontrolled inflammation is associated with neurodegenerative conditions in central nervous system tissues, including the retina and brain. We previously found that the neural retina (NR) plays an important role in retinal immunity. Tumor necrosis factor Receptor-Associated Factor 3 (TRAF3) is a known immune regulator expressed in the retina; however, whether TRAF3 regulates retinal immunity is unknown. We have generated the first conditional NR-Traf3 knockout mouse model (Chx10-Cre/Traf3 $\left.{ }^{\mathrm{f} / \mathrm{f}}\right)$ to enable studies of neuronal TRAF3 function. Here, we evaluated NRTraf3 depletion effects on whole retinal TRAF3 protein expression, visual acuity, and retinal structure and function. Additionally, to determine if NR-Traf3 plays a role in retinal immune regulation, we used flow cytometry to assess immune cell infiltration following acute local lipopolysaccharide (LPS) administration. Our results show that TRAF3 protein is highly expressed in the NR and establish that NR-Traf3 depletion does not affect basal retinal structure or function. Importantly, NR-Traf3 promoted LPS-stimulated retinal immune infiltration. Thus, our findings propose NR-Traf3 as a positive regulator of retinal immunity. Further, the NR-Traf3 mouse provides a tool for investigations of neuronal TRAF3 as a novel potential target for therapeutic interventions aimed at suppressing retinal inflammatory disease and may also inform treatment approaches for inflammatory neurodegenerative brain conditions.

Keywords: neural retina; Traf3; immunity; inflammation; neurodegeneration; central nervous system; vision; lipopolysaccharide

\section{Introduction}

Immune responses have evolved to defend host tissues from foreign invaders. These responses are particularly important for maintenance of post-mitotic central nervous system (CNS) neurons in the brain and retina following infection or injury, as neuronal cells lack the ability to regenerate after cell death. However, prolonged overactivation of these immune processes can result in neurotoxicity, which commonly occurs during inflammatory neurodegenerative disease progression and ultimately results in neuronal cell death. Unfortunately, neuroinflammatory regulation is not well understood and is further confounded by the complex nature of immune privilege mechanisms present in neuronal tissues [1]. Often considered a "window to the brain", the retina is an accessible extension of the CNS that is more amenable to less-invasive in vivo observations $[2,3]$.

Thus, in addition to advancing our knowledge regarding blindness-causing neurodegenerative diseases, retinal mouse models also have the potential to inform neurodegenerative brain research. 
The retina functions to receive, convert, and transmit light information in the form of electrical and chemical signals that are interpreted by the brain for visual processing [4]. Retinal neurons, including photoreceptor (i.e., rods and cones), horizontal, bipolar, amacrine, and ganglion cells participate in a signaling cascade (i.e., phototransduction) that transmits this translated information to the brain for further visual interpretation $[5,6]$. Chronic retinal neurodegeneration, thus, leads to vision loss and, eventually, complete blindness $[7,8]$. In addition to the aforementioned neuronal populations, many other cell types are present in the retina and support healthy neuronal function, including providing immune protection in response to infection and/or injury. Microglia, astrocytes, and retinal pigment epithelia (RPE) have long been known to play important roles in retinal immune homeostasis, and cells of the retinal vasculature modulate the highly-selective permeability of the blood-retina barrier (BRB) during inflammation [9-12]. Recently, we found that the neural retinal (NR) compartment, which is comprised of all retinal neurons and Müller glial cells, also plays an important role in retinal immune regulation [13].

In a previous study, we identified Tumor necrosis factor Receptor-Associated Factor 3 (TRAF3) as a novel potential immune regulator associated with retinal membrane fractions [13]. TRAF3 has been well established as a cytosolic and nuclear adapter protein that directly interacts with cell surface receptors to regulate a wide variety of signaling pathways and cellular processes [14-18]. Global-Traf3 depletion in mice results in neonatal lethality [19]. However, conditional-Traf3 knockout mouse models have been successfully generated and, in conjunction with cell culture models, have provided valuable insights regarding additional cell-specific TRAF3 functions, including cell survival, metabolic regulation, growth, proliferation, and immune modulation [20-26]. Notably, gene and protein databases report high Traf3 expression in the brain and retina [27]. However, studies regarding TRAF3 function in neuronal tissues have only recently begun to be investigated [24,28,29]. Further, to our knowledge, no retinal-specific mouse models have yet been developed and retinal TRAF3 function has not been explored. Thus, the goals for this study were (1) to develop a novel genetic NR-Traf3 depletion model, (2) to investigate potential basal effects of NR-Traf3 ablation on retinal tissue architecture and function, and (3) to determine if NR-Traf3 plays a functional role in retinal immunity. Our study, thus, also evaluates the NR-Traf3 mouse as a useful model for assessing in vivo neuronal-specific TRAF3 function.

\section{Materials and Methods}

\subsection{Materials}

Information for the proceeding reagents and materials is as follows: n-octyl- $\beta$-Dglucopyranoside (Cat\# 494459), Triton X-100 (Cat\# X100), NaCl (Cat\# S3014), EDTA (Cat\# 324506), complete mini protease inhibitor cocktail tablets (Cat\# 11836170001), bovine serum albumin (BSA; Cat\# A7906), glycerol (Cat\# G5516), and lipopolysaccharides from Salmonella enterica serotype typhimurium (LPS; Cat\# L2262) were purchased from MilliporeSigma (Burlington, MA, USA). The Pierce BCA protein assay kit (Ref 23227), Invitrogen (Waltham, MA, USA) Novex WedgeWell 8\% Tris-Glycine Gels (1.0 mm × 12 well; Ref XP00082BOX), BenchMark Prestained Protein Ladder (Ref 10748-010), XCell SureLock Mini-Cell gel electrophoresis system (Ref EI0001), 10× Novex Tris-Glycine SDS Running Buffer (Cat\# LC2675-5), and SuperSignal West Dura Extended Subtrate (Ref 34076) were purchased from ThermoFisher Scientific (Waltham, MA, USA). Nitrocellulose membrane (0.45 $\mu \mathrm{m}$; Cat\# 1620115), Mini Trans-Blot Cell system (Cat\# 1703930), and PowerPac Basic power supply (Cat\# 1645050) were from BioRad (Hercules, CA, USA). Normal horse serum blocking solution (S-2000-20) was purchased from Vector Laboratories (Burlingame, CA, USA).

Antibodies for Western blotting were obtained as follows: primary rabbit polyclonal anti-TRAF3 was purchased from Novus Biologicals (\#NBP1-86639; Littleton, CO, USA). Primary mouse monoclonal anti-Actin was purchased from ThermoFisher (\#MA1-744). Donkey anti-rabbit (NA934V) and sheep anti-mouse (NA931V) horseradish peroxidase (HRP)-linked secondary antibodies were purchased from Global Life Sciences Solutions 
(Marlborough, MA, USA). Antibodies for retinal immunofluorescence were obtained as follows: primary mouse monoclonal anti-Glutamine Synthetase was purchased from MilliporeSigma (\#MAB302, clone GS-6). Primary rat monoclonal mouse anti-CD31 was purchased from Dianova (\#DIA-310; Hamburg, Germany). Alexa Fluor 488-conjugated goat anti-mouse secondary antibody (A-11001) was purchased from ThermoFisher. Alexa Fluor 647-conjugated donkey anti-rat secondary antibody (Code: 712-605-150) was purchased from Jackson ImmunoResearch (West Grove, PA, USA). Antibodies for flow cytometry were obtained as follows: Pacific Blue-conjugated anti-mouse CD45 (clone 30-F11), PEconjugated anti-mouse GR1 (Ly6G/Ly6C; clone RB6-8C5), Spark Blue 550-conjugated anti-mouse CD45 (clone 30-F11), APC-Cy7-conjugated anti-mouse Ly6C (clone HK1.4), PerCP-Cy5.5-conjugated anti-mouse Ly6G (clone 1A8), PE-conjugated anti-mouse/human CD11b (clone M1/70), PE-Cy7-conjugated anti-mouse CD115 (CSF-1R; clone AFS98), APC-conjugated anti-mouse F4/80 (clone BM8), BV650-conjugated CCR2 (CD192; clone SA203G11), and BV605-conjugated MHC II (clone M5/114.15.2) were all purchased from Biolegend, San Diego, CA, USA. The Zombie Aqua fixable viability reagent for 7-color flow cytometry was also purchased from Biolegend, San Diego, CA, USA.

\subsection{Chx10-Traf3 KO Model}

All animal procedures were approved by the University of Oklahoma Health Sciences Center Institutional Animal Care and Use Committee (OUHSC IACUC) and follow the Association for Research in Vision and Ophthalmology (ARVO) Statement for the Use of Animals in Ophthalmic and Visual Research. Neural retinal-specific Traf3 knockout (NR-Traf3 KO) animals were generated via Cre-Lox technology by breeding mice carrying Chx10-promoter-driven Cre recombinase with Traf3-floxed mice provided by G.A. Bishop (Figure 1a) [20]. Expression of Chx10-Cre (stock\#: 005105, The Jackson Laboratory, Bar Harbor, ME) occurs specifically in neural retinal progenitor cells, resulting in recombination in retinal neurons (photoreceptor, bipolar, and ganglion cells), and Müller glial cells (Figure 1b) $[13,30,31]$. Recombination within the Traf3 gene occurs via Lox P sites located upstream of exon 1 and downstream of exon 2 [20].

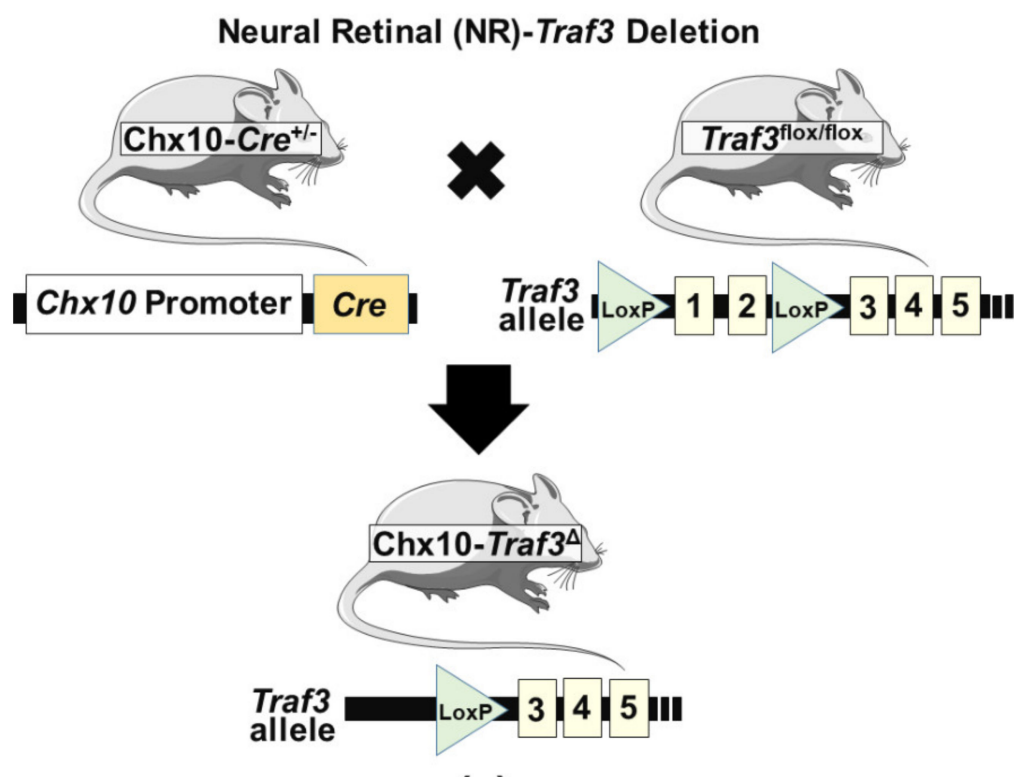

(a)

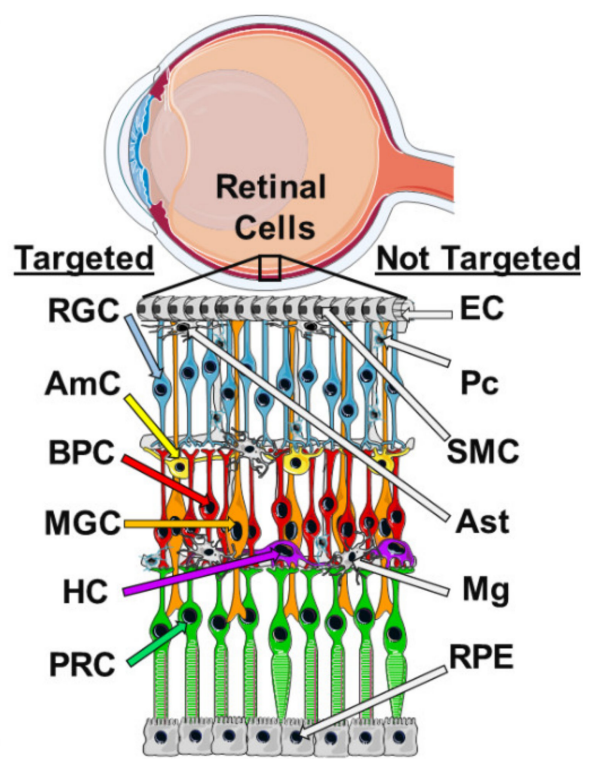

(b)

Figure 1. Schematic diagram showing neural retinal (NR)-specific genetic deletion of Traf3 in the Chx10-Cre/Traf3 flox/flox (NR-Traf3 KO) mouse model. (a) Breeding strategy for NR-specific Traf3 depletion. (b) Retinal tissue diagram outlining resident retinal cell populations targeted (and not targeted) for Traf3 deletion using the NR-Traf3 KO model. Targeted: RGC, retinal ganglion cell; AmC, amacrine cell; BPC, bipolar cell; MGC, Müller glial cell; HC, horizontal cell; PRC, photoreceptor cell. Not targeted: EC, endothelial cell; Pc, pericyte; SMC, smooth muscle cell; Ast, astrocyte; Mg, microglia; RPE, retinal pigment epithelia. 


\subsection{Whole Retinal Tissue Lysis}

Whole retinal protein extracts were obtained via brief sonication of one retina $(\sim 6-7 \mu \mathrm{g})$ per sample in $65 \mu \mathrm{L} 2 \times$ lysis buffer $(120 \mathrm{mM}$ octyl glucoside, $2 \%$ Triton X-100, $20 \mathrm{mM}$ Tris- $\mathrm{HCl}, \mathrm{pH}$ 7.4, $200 \mathrm{mM} \mathrm{NaCl}, 1 \mathrm{mM}$ EDTA) containing a protease inhibitor cocktail tablet (Roche). Lysates were cleared by 10 -min centrifugation at $13 \mathrm{~K} \mathrm{rpm}$, at $4{ }^{\circ} \mathrm{C}$. Protein concentration was determined via BCA assay, using bovine serum albumin standard, and samples were diluted in $6 \times$ Laemmli buffer and stored at $-80^{\circ} \mathrm{C}$ until further use.

\subsection{Western Blotting}

Whole retinal tissue protein separation was achieved via SDS-PAGE. Proteins were then transferred to nitrocellulose for immunoblotting with the following primary antibodies and dilutions: rabbit polyclonal anti-TRAF3 (1:500; Novus Biologicals, Cat\# NBP1-86639), mouse monoclonal anti-Actin (1:1000; ThermoFisher, \#MA1-744). Immunoreactivity was detected using species-appropriate horseradish peroxidase (HRP)-conjugated secondary antibodies (1:5000 for TRAF3 and 1:10,000 for Actin; GE Healthcare, Cleveland, OH, USA). Western blots were imaged via HRP chemiluminescent detection (Azure Biosystems, Inc.; Dublin, CA, USA) and densitometric analyses were performed using Image Studio Lite software (LI-COR Biosciences; Lincoln, NE, USA).

\subsection{Immunohistochemistry and Confocal Microscopy}

After adult mice were euthanized, whole eye globes were fixed in Prefer fixative (Anatech, Ltd., Battlefield, MI, USA), embedded in paraffin, and cut into $5 \mu \mathrm{m}$ sections. Deparaffinization was performed via successive incubations using the following protocol: $3 \times$ for 5 min each in fresh xylene, $2 \times$ for 3 min each in $100 \%$ ethanol, and $1 \times$ for $3 \mathrm{~min}$ in $95 \%$ ethanol. Sections were then rinsed with deionized water $3 \times$, were permeabilized with $1 \%$ Triton X-100 in PBS, then incubated with blocking solution (10\% normal horse serum, $0.1 \%$ Triton $X-100$, in 1 XPBS) for one hour prior to immunohistochemistry, which was performed as previously described using either hematoxylin and eosin (H\&E) staining or the following antibodies: primary mouse monoclonal anti-Glutamine Synthetase (1:5000, MilliporeSigma \#MAB302, clone GS-6) and rat monoclonal anti-mouse CD31 (1:100, Dianova \#DIA-310). Immunoreactivity was detected with species-appropriate Alexa Fluor-conjugated secondary antibodies (1:500; goat anti-mouse 488, donkey anti-rat 647). Images of immunostained retinal tissue sections were captured using an Olympus FV1200 laser scanning confocal microscope and visualized using FluoView software (Olympus). Pseudocolors were assigned to images as follows: Glutamine Synthetase, green; CD31, red.

\subsection{Optokinetic Tracking (OKT)}

Visual acuity was assessed via non-invasive observations of unrestrained mouse behavioral (head turning) responses to a rotating visual gradient as described by Prusky et al. [32]. Briefly, systematic increases in spatial frequencies were used to determine maximum visual thresholds for both left and right eyes in all mice (OptoMotry; CerebralMechanics, Lethbridge, AB, Canada). For each mouse, two maximum threshold measurements per eye were recorded and averaged.

\subsection{Optical Coherence Technology (OCT)}

Spectral domain optical coherence technology/tomography (SD-OCT; Bioptigen; Durham, NC, USA), in conjunction with InVivoVue Diver software (Version 2.4) analysis, was used for in vivo assessment of retinal structure. After determining that no apparent anomalous retinal defects were present, quantitative retinal layer thickness measurements for each animal were calculated individually for the right eye (RE) and left (LE) eye of each animal. Automated total and layer-specific retinal thickness values were calculated via manual determination of 10 retinal boundaries, which defined 9 distinct retinal tissue layers. These retinal tissue layers were obtained for 8 "inner" and "outer" retinal zones (relative to the central optic nerve head $(\mathrm{ONH})$ ) comprising Superior, Temporal, Inferior, 
and Nasal positions of each retina. Mirrored retinal zone measurements for the left and right eyes of each animal were then averaged to obtain a single measurement, per animal, for each retinal zone location. Following determination of averaged individual mouse retinal layer measurements, group data were then combined to assess potential genotypespecific differences in total retinal thickness as well as for each of the smaller encapsulated subdivisions and individual retinal layers.

\subsection{Electroretinography (ERG)}

ERGs were recorded as described previously [33,34]. Briefly, overnight dark-adapted mice were anesthetized with ketamine $(100 \mathrm{mg} / \mathrm{kg})$ and xylazine $(10 \mathrm{mg} / \mathrm{kg})$, pupils were dilated with $0.5 \%$ atropine and $2.5 \%$ phenylephrine, gold wire electrodes were positioned on corneas, a reference electrode was placed inside the mouth, and a ground electrode was places in the tail. Rod-driven responses were assessed by presenting increasing scotopic stimuli ( -3.7 to $2.6 \log$ scotopic candela $(\mathrm{cd}) \times \mathrm{s} / \mathrm{m}^{2}$ ) via a Colordome Espion ERG recording system (Diagnosys, Lowell, MA, USA). Intensity response relationships for a- and b-wave were fit using the GraphPad Prism Michaelis-Menten equation in order to calculate physiological maximum amplitudes (i.e., Vmax) [33,34].

\subsection{Endotoxin-Induced Uveitis Model}

Intravitreal injection was used to locally administer $1 \mu \mathrm{g}$ LPS (Salmonella typhimurium; Sigma) in order to induce ocular inflammation as described previously [13]. Briefly, mice anesthetized with intraperitoneal ketamine $(100 \mathrm{mg} / \mathrm{kg}) /$ xylazine $(10 \mathrm{mg} / \mathrm{kg})$ injection received $1 \mu \mathrm{L}$ contralateral injections of either saline $(1 \times$ PBS) vehicle or LPS (diluted in $1 X$ PBS vehicle), via a $10 \mu \mathrm{L}$ glass syringe (Hamilton) with a 33-gauge needle, into the vitreous chamber of each eye. To ensure minimal damage to ocular tissues, the eye was gently proptosed and held in place by clasping the surrounding eyelids during injections. Whole retinal tissue was harvested, using the "Winkling" method, $24 \mathrm{~h}$ after immune induction for downstream assessment of innate immune infiltration using flow cytometry [35-37].

\subsection{Flow Cytometry}

Processing and staining of retina tissue for flow cytometry was performed as previously described, but with some minor modifications [13]. Briefly, following whole animal perfusion, extracted retinal tissue was minced and digested with liberase TL for $20 \mathrm{~min}$ at $37^{\circ} \mathrm{C}$. The cell suspension from digested tissue was filtered through $40 \mu \mathrm{m}$ cell strainer and washed with PBS supplemented with $2 \%$ FCS and $2 \mathrm{mM}$ EDTA (called herein as Staining Buffer or SB). Fc receptors were blocked using anti-mouse CD16/32 (Fc-block; Invitrogen) at $4{ }^{\circ} \mathrm{C}$ for $10 \mathrm{~min}$. For 3-color flow cytometry, cells were stained with the following antibodies: Pacific Blue-conjugated anti-mouse CD45 (clone 30-F11), PE-conjugated anti-mouse GR1 (Ly6G/Ly6C; clone RB6-8C5) and APC-conjugated anti-mouse F4/80 (clone BM8). For 7-color flow cytometry, cells were first stained with cell viability dye (Zombie Aqua) for $15 \mathrm{~min}$ at room temperature followed by washing with SB. Finally, the cells were Fc-blocked $\left(10 \mathrm{~min}\right.$ at $4{ }^{\circ} \mathrm{C}$ ) and stained ( $25 \mathrm{~min}$ on ice) with anti-mouse antibody cocktail containing Spark Blue 550-conjugated anti-mouse CD45 (clone 30-F11), APC-Cy7conjugated anti-mouse Ly6C (clone HK1.4), PerCP-Cy5.5-conjugated anti-mouse Ly6G (clone 1A8), PE-conjugated anti-mouse/human CD11b (clone M1/70), PE-Cy7-conjugated anti-mouse CD115 (CSF-1R; clone AFS98), APC-conjugated anti-mouse F4/80 (clone BM8), BV650-conjugated CCR2 (CD192; clone SA203G11), and BV605-conjugated MHC II (clone M5/114.15.2). For 3-color flow cytometry analysis, data were acquired using a MacsQuant flow cytometer (Miltenyi Biotec, Auburn, CA, USA). Events were gated using forward and side scatter, along with sequential gating to distinguish infiltrating cells as previously described [38]. For 7-color flow cytometry analysis, sample data were acquired using 4-laser spectral flow cytometer Aurora (Cytek Biosciences, Fremont, CA, USA) containing 16 violet, 14 blue, 10 yellow-green and 8 red channels (4L-16V-14B-10YG-8R). Each fluorochrome peaked in a separate channel and a general spectra pattern was validated based on the 
references provided by Cytek Biosciences (online resources: Cytek Full Spectrum Viewer; website: https://spectrum.cytekbio.com/ (accessed on 11 March 2021). Compensation for spectral unmixing was performed using reference controls and unmixing wizard integrated in SpectroFlo software. The gating strategy was established based on running FMO (Fluorescence Minus One) retina samples. Acquired data for all samples were exported as FCS files and analyzed with FlowJo software version 10.7.1 (BD Biosciences, Ashland, OR, USA) as previously described [13].

\subsection{Other Methods}

Numerical data are represented as the mean \pm standard error of the mean (SEM). Statistically significant differences among groups were determined using GraphPad Prism (Version 8.1.2) Student's $t$-test, one-way analysis of variance (ANOVA), or two-way ANOVA where appropriate and are described in corresponding figure legends. Statistically significant data were defined as having a $p$-value $<0.05$. For functional studies, Chx10-Cre ${ }^{+}$ control and Traff flox/flox controls were pooled as "NR-Traf3 WT," as we did not observe any differences between these groups. For Western blot data, Chx10-Cre ${ }^{-} /$Traffflox/flox animals were used as controls.

\section{Results}

\subsection{The Majority of Retinal TRAF3 Protein Expression Is in the Neural Retinal (NR) Compartment}

We and others have previously demonstrated that the Chx10-Cre/Lox genetic knockout model is an effective tool for targeted neural retinal-specific gene depletion [13,30,31]. Here, we generated conditional neural retinal-Traf3 knockout mice (NR-Traf3 KO) by breeding our Chx10-Cre mouse line with a Traf $3^{\text {flox/flox }}$ mouse line (Figure 1a). This NR-Traf3 KO is characterized by Cre-mediated recombination of Traf3 exons 1 and 2. As Chx10 (also known as Ceh-10 Homeodomain-Containing Homolog/Vsx2, Visual System Homeobox 2 ) is actively expressed in all neural retinal progenitor cells during development, cell-specific recombination occurs in all retinal neurons as well as supporting Müller glial cells. Figure $1 \mathrm{~b}$ provides a simplified diagram showing Chx10-targeted retinal cell types (and their localization within the retina) as well as retinal cell populations not targeted by the NR-Traf3 KO model.

To assess the effect of NR-Traf3 depletion on whole retinal tissue TRAF3 protein expression, we performed Western blot analysis of retinal tissue extracts in adult NR-Traf3 WT and $\mathrm{KO}$ mice (Figure 2). We found that NR-Traf3 $\mathrm{KO}$ retinas exhibited a $74.6 \%$ reduction in total retinal TRAF3 protein compared to WT animals (Figure 2a). In a second cohort, we also observed a dose-dependent decrease in TRAF3 protein where heterozygous and homozygous NR-Traf3 mice displayed $30.7 \%$ and $62.1 \%$ decreases, respectively, compared to WT controls (Figure S1a). Thus, our data suggest that the neural retinal TRAF3 pool accounts for the majority of TRAF3 expression in the retina and that the NR-Traf3 KO model provides a novel tool for assessing the importance of Traf3 in the neural retinal (NR) compartment. To evaluate the specificity of Chx10-Cre-mediated recombination, we examined TRAF3 expression in brains of NR-Traf3 KO mice and WT littermate controls and found no difference in TRAF3 expression (Figure 2b). Interestingly, we noted that the retina exhibited relatively higher TRAF3 levels compared to brain neuronal tissue (per $\mu \mathrm{g}$ total protein) as approximately $2.4 \times$ the amount of brain protein extract was required to detect TRAF3 within the linear range of the assay. We observed no differences in genotype-dependent body weights within male or female groups, and there were no sex- or genotype-dependent differences in brain or retinal tissue weights (Figure S1b). 
(a)

Retina

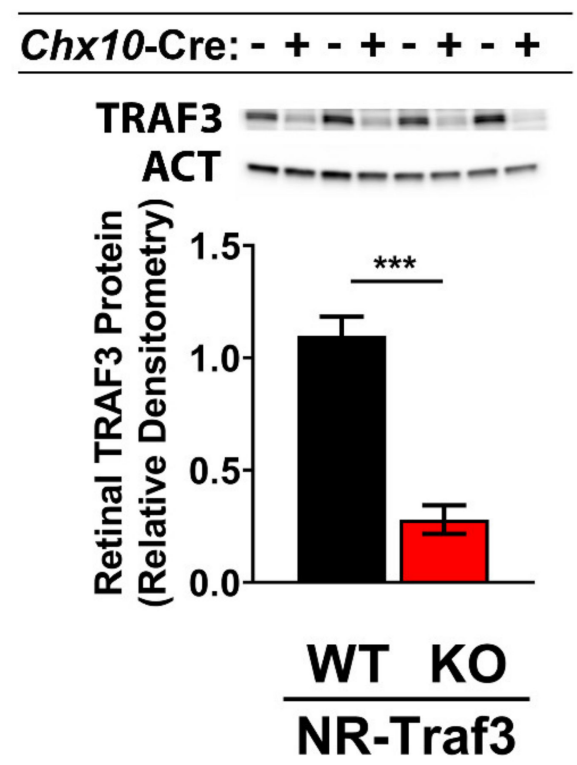

(b) Brain

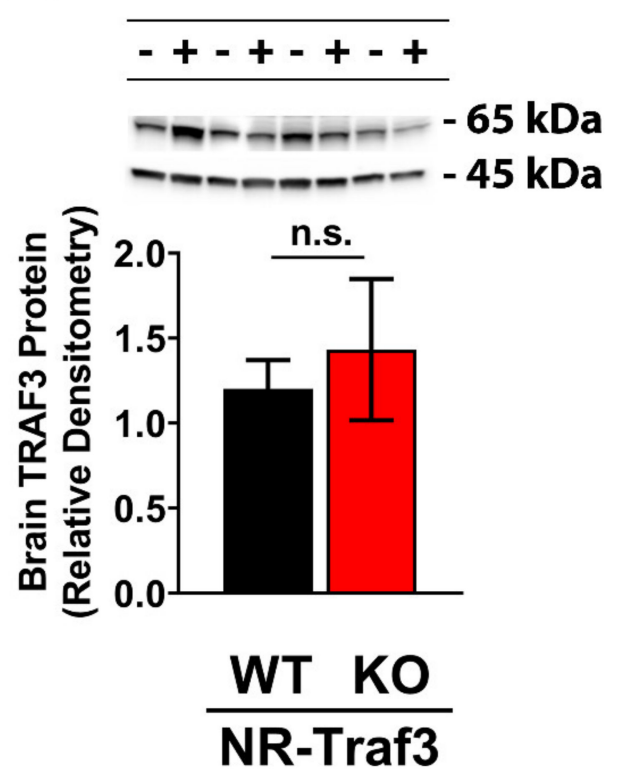

Figure 2. NR-Traf3 KO mice exhibit significant and specific reductions in whole retinal tissue TRAF3 protein. TRAF3 Western blot data and corresponding quantitative densitometry analysis for neuronal whole retinal (a) and brain (b) tissues extracts, respectively. $\beta$-actin was used as loading control and for normalization of TRAF3 protein expression. To detect protein within the linear range of the assay, protein loads were as follows: retinal TRAF3, $25 \mu \mathrm{g}$; brain TRAF3, $60 \mu \mathrm{g}$; retinal/brain $\beta$-actin, $10 \mu \mathrm{g}$. Chx10-Cre negative (-) and positive (+) expression indicate NR-Traf3 WT and KO mice, respectively. Data are the mean \pm SEM and were analyzed via Student's $t$-test $(* * *<<0.001 ; n=4)$.

\subsection{Neural Retinal Traf3 Depletion Does Not Adversely Affect Basal Retinal Structure or Function}

Global Traf3 depletion results in neonatal lethality around P10 in mice [19]. However, several viable non-ocular conditional Traf3 $\mathrm{KO}$ models have been successfully generated $[17,21,24]$. To our knowledge, we have developed the first ocular model of conditionalTraf3 depletion and have shown that TRAF3 protein is highly expressed in the NR compartment of retinal tissue (Figure 2). Thus, before investigating the potential role of TRAF3 in retinal immunity, we first wanted to identify whether NR-Traf3 ablation had any impact on basal retinal tissue development, structure, and/or function prior to immune challenge.

\subsubsection{Retinal Tissue Structure and Patterning}

To assess potential overall defects of NR-Traf3 $\mathrm{KO}$ on retinal tissue development and/or morphology, we used in vivo spectral domain optical coherence technology/ tomography (SD-OCT) to measure total retinal thickness (in eight distinct retinal zones) as defined by the distance from the inner limiting membrane of the retinal neural fiber layer (RNFL) to the outermost boundary of Verhoeff's membrane located in the RPE (Figures 1 and $3 a$ ). 


\section{(a) Retinal Zones}

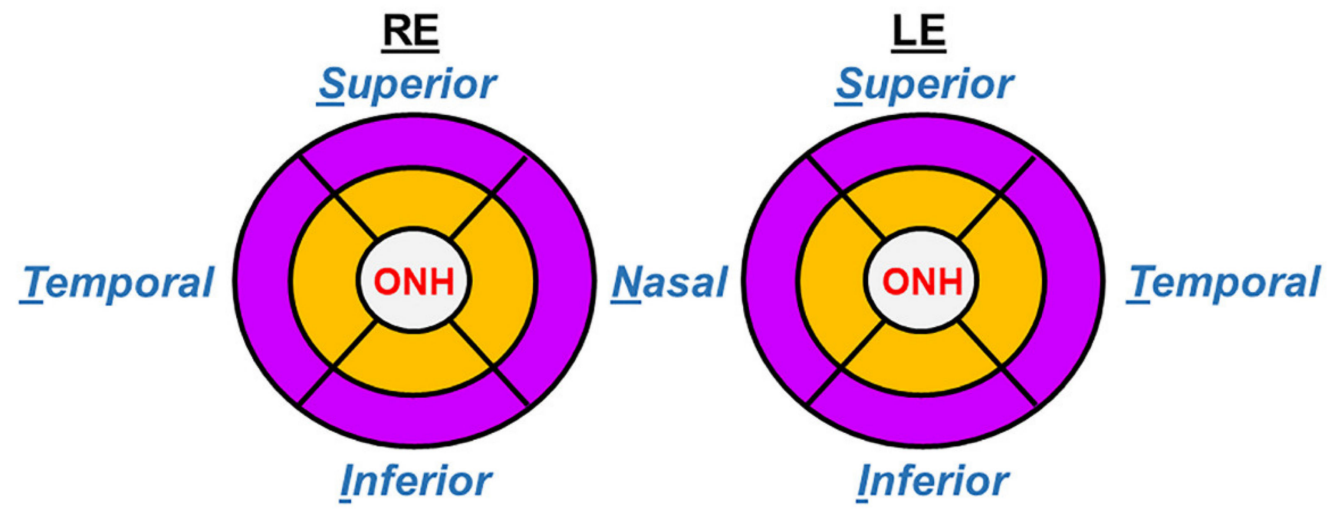

\section{Retinal Thickness}
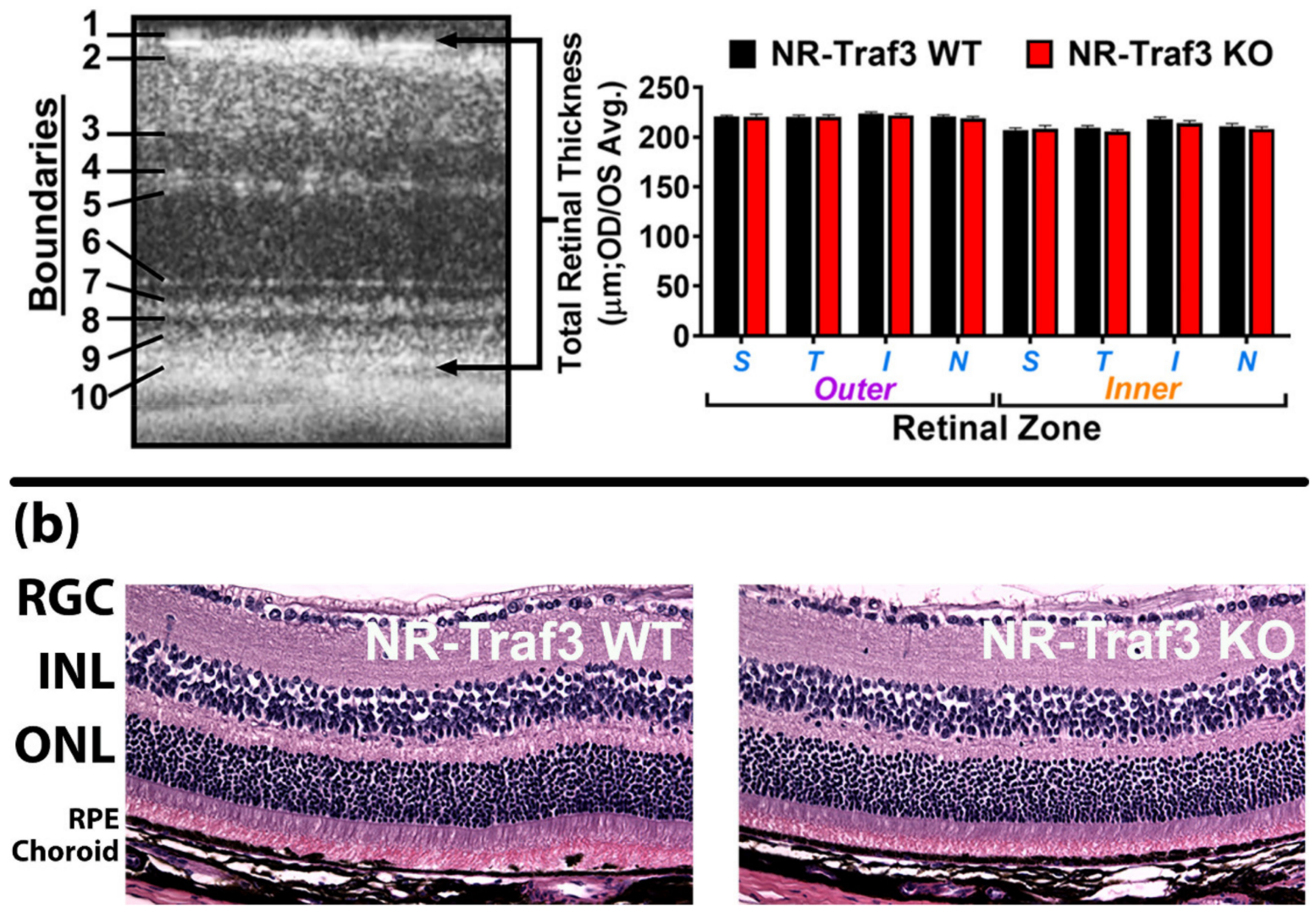

Figure 3. NR-Traf3 KO mice display normal retinal structure and lamination. (a) Diagram showing distinction of outer (purple) and inner (orange) retinal regions with further separation into superior, temporal, inferior, and nasal zones. Total retinal thickness (TRT) was measured for all 8 retinal zones for both eyes and was defined as the total distance (in $\mu \mathrm{m}$ ) between boundaries 1 (inner limiting membrane) and 10 (Verhoeff's membrane), which is illustrated in the example SD-OCT image. Additional boundaries used to measure thicknesses of subdivisions and individual sublayers within the TRT are also noted in the example SD-OCT image (see also Table S1). Numerical data for TRT and retinal sublayers are provided in Table S1. (b) representative H\&E-stained retinal sections showing normal lamination of retinal layers: RE, right eye; LE, left eye; ONH, optic nerve head; S, superior; T, temporal; I, inferior; N, nasal; RGC, retinal ganglion cell layer; INL, inner nuclear layer; ONL, outer nuclear layer; RPE, retinal pigment epithelial layer. TRT data are represented as the mean \pm SEM. ( $n=6$; Student's $t$-tests for each retinal zone were not significant).

There was no difference in total retinal thickness for any of the eight retinal zones when comparing NR-Traf3 WT and KO retinas (Figure 3a). Additionally, we found no 
differences within either the smaller inner, middle, and outer retinal subdivisions (IRT, MRT, and ORT, respectively) or the individual sublayers that comprise these subdivisions of the retina (Table S1). We also performed SD-OCT in NR-Traf3 heterozygous (HET) animals (Table S1). Interestingly, we did find small, but statistically significant differences between WT and HET INL of the inner nasal retinal zone IRL subdivision, which also affected the TRT. However, there were no statistical differences found for inner nasal retinal zone IRL RNFL or IPL sublayers individually. Thus, taken together, our SD-OCT results suggest that NR-Traf3 depletion does not affect overall retinal structure, and that NR-Traf3 $\mathrm{KO}$ mice exhibit normal retinal cellular arrangement and regular tissue configuration under basal conditions.

To validate that there was no overall effect of NR-Traf3 depletion on retinal architecture, we analyzed H\&E-stained adult retinal paraffin sections (Figure 3b). We observed normal lamination of all three neuronal layers (i.e., RGC, INL, and ONL), as well as intact RPE and choroidal vascular layers (Figure $3 \mathrm{~b}$ and Figure S2). Vascular cross sections for all three retinal vascular layers were also present (Figure S2) and no edema was observed. Additionally, photoreceptor inner and outer segments could be visualized and appeared to have typical morphology. Müller glial development and differentiation also appeared normal as evidenced by immunofluorescent Glutamine Synthase (GS) detection in retinal paraffin sections (Figure S2).

\subsubsection{Visual and Retinal Function}

To measure overall visual function, we used optokinetic tracking (OKT) to evaluate spatial vision as a measure of visual acuity. Using a virtual optomotor system, we measured the behavioral temporal-to-nasal optokinetic reflex in NR-Traf3 WT and KO mice for each eye (left $=\mathrm{LE}$, right $=\mathrm{RE}$ ) [32]. We found no genotype-dependent differences in visual acuity for NR-Traf3 KO animals compared to WT littermates (Figure 4; LE, WT: $0.397 \pm 0.011$, KO: $0.386 \pm 0.012$; RE WT: $0.398 \pm 0.013, \mathrm{KO}: 0.386 \pm 0.029)$, suggesting that overall visual function was not impaired.

To measure retinal function, we used scotopic electroretinography (ERG) to assess NR-Traf3 effects on rod photoreceptor-derived scotopic electrical stimuli for a range of flash intensities (Figure 5). We first generated response amplitude curves for both aand b-wave responses (Figure 5a), which were then transformed into Vmax histograms for physiological interpretation (Figure $5 b$ ). We did not find any genotype-dependent differences in a-wave responses for any of the flash intensities tested, which corresponded to no difference in a-wave Vmax between groups (WT: $292.9 \pm 23.4$; KO: $264.8 \pm 61.8$ ). For b-wave intensity, we found a reduction in the NR-Traf3 KO response at a flash intensity of $-1 \log \mathrm{cd} \times \mathrm{s} / \mathrm{m}^{2}$; however, this difference was not sufficient to alter the physiological b-wave Vmax compared to WT controls (WT: 602.2 \pm 38.4; KO: $511.2 \pm 103.2$ ). Thus, we conclude that NR-Traf3 does not significantly affect retinal function under basal conditions. 


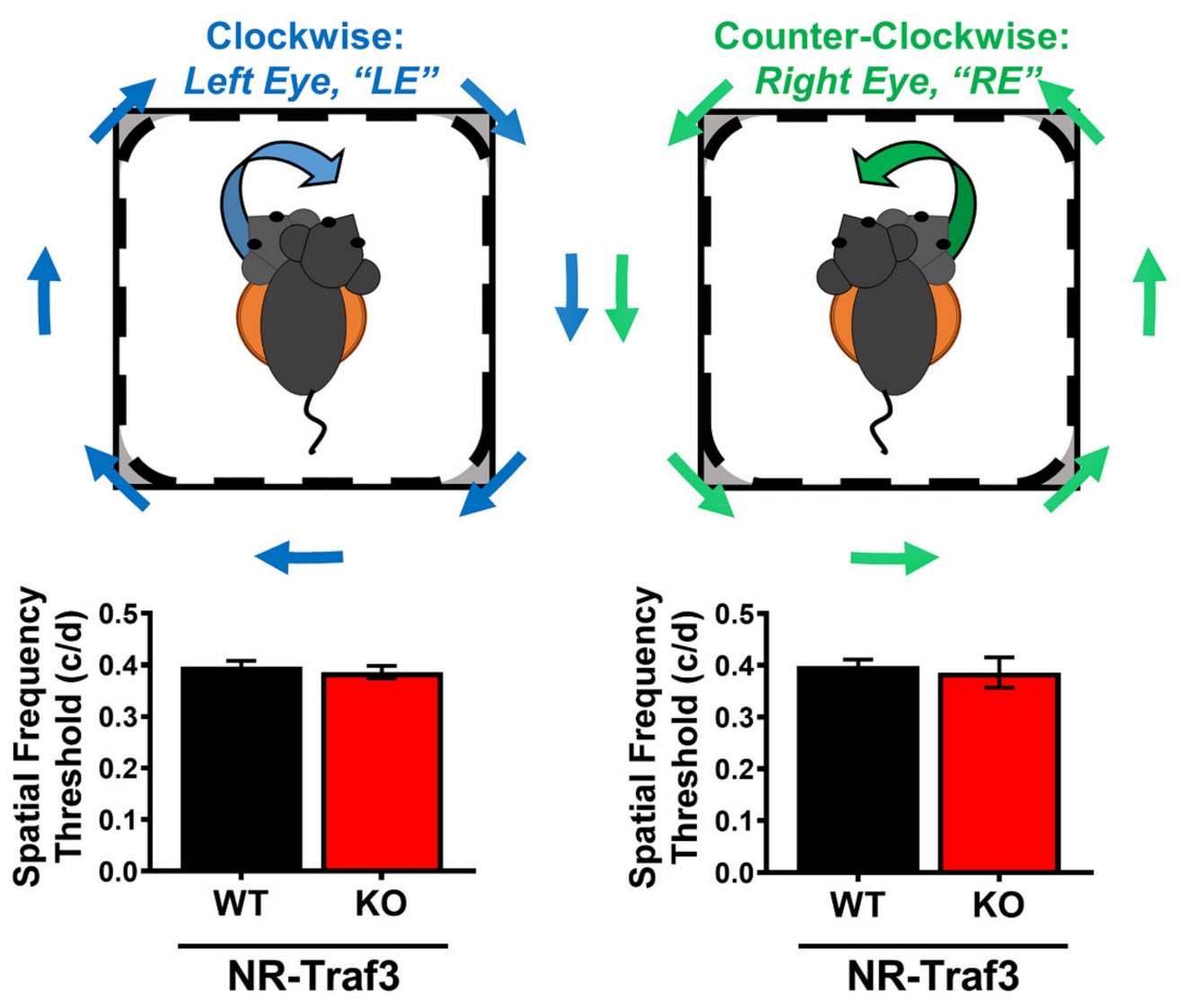

Figure 4. NR-Traf3 depletion does not affect basal visual acuity. Schematic (top) shows virtual cylinder grating rotation direction for left eye (LE, clockwise) and right eye (RE, counter-clockwise) spatial frequency determinations. Visual acuity data for left and right eyes show no difference in visual thresholds between NR-Traf3 WT and KO animals. ( $n=5-12$; Student's $t$-test, not significant).

(a) Intensity Response

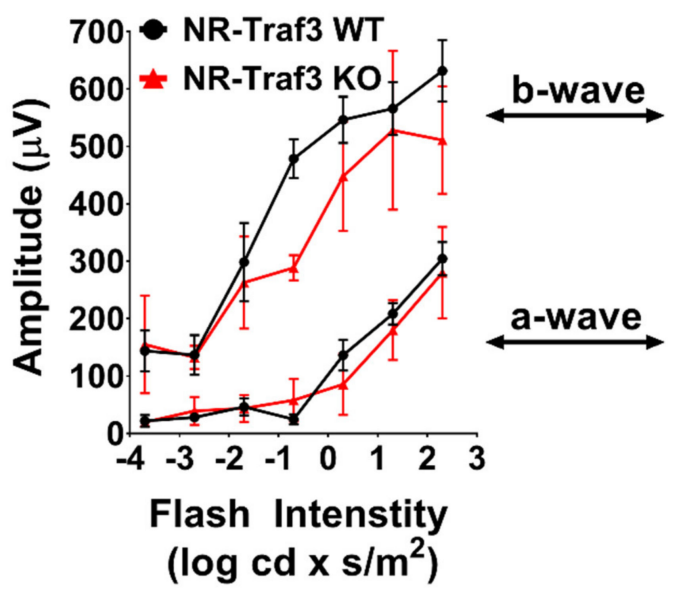

(b) $\quad \max$

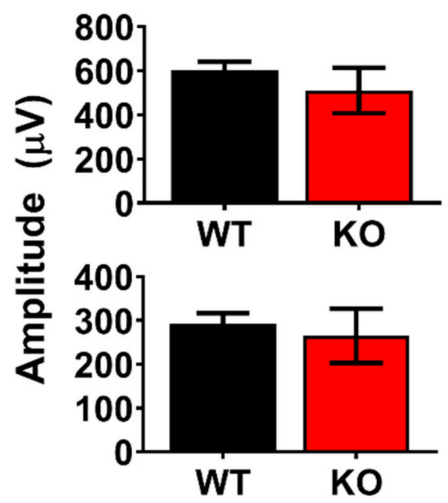

Figure 5. NR-Traf3 KO mice exhibit normal basal retinal function. Scotopic electroretinography (ERG) data represent electrical response curves for (a) increasing flash intensity exposures. Response curves were used to determine (b) maximum amplitudes for rod photoreceptor-derived a-wave and b-wave responses. NR-Traf3 $\mathrm{KO}$ resulted in decreased b-wave amplitude at $-1 \log \mathrm{cd} \times \mathrm{s} / \mathrm{m}^{2}$; however, there was no physiological difference in Vmax for either a- or b-waves between NR-Traf3 WTs and Kos. ( $n=3-7$; Student's $t$-test, not significant). 


\subsection{Neural Retinal Traf3 Promotes the Retinal Immune Response to Endotoxin-Mediated Inflammatory Activation}

Intravitreal LPS injection (LPS ${ }^{\text {ivt }}$ ) has been established as an effective model for inducing acute murine ocular inflammatory leukocyte infiltration [39]. To initially assess whether NR-Traf3 depletion affects retinal immune cell infiltration, we first performed a 3-color flow cytometry experiment on NR-Traf3 WT and KO retinas $24 \mathrm{~h}$ after LPSivt induction (Figure S3). We observed an expected dramatic increase in the number of "Total Leukocytes" (CD45 ${ }^{+}$cells) from NR-Traf3 WT retinas following LPS ${ }^{\text {ivt }}$ injection $(66.2 \%$ increase; PBS: $2455 \pm 512$ vs. LPS: $7270 \pm 1490 ; p=0.001$ ). Immune cell numbers also increased in NR-Traf3 KOs with LPS ${ }^{\text {ivt }}$ treatment, though this did not statistically differ from that of contralateral PBS controls (37.5\% increase; PBS: $1959 \pm 525$ vs. LPS: $3137 \pm 604$; $p=0.3571$ ). Furthermore, LPSivt-treated NR-Traf3 KOs retinas exhibited significantly decreased infiltrate compared to their LPS ${ }^{\text {ivt }}$-treated NR-Traf3 WT littermates (43.1\% of WT response; NR-Traf3 WT LPS: $7270 \pm 1490$ vs. KO LPS: $3137 \pm 604 ; p<0.01)$, suggesting that NR-Traf3 promotes retinal inflammation. Likewise, NR-Traf3 KO retinas exhibited an LPS-dependent reduction in GR $1^{+} F 4 / 80^{-}$cells (WT PBS: $1055 \pm 303$, LPS: $4553 \pm 765$ vs. KO PBS: $640 \pm 298$, LPS: $1176 \pm 413 ; p<0.0001$, LPS WT vs. KO), which accounted for the majority of total infiltrating leukocytes $\left(\mathrm{CD}^{+} 5^{+}\right.$cells) and suggested that most pervading cells harbored a "Polymorphonuclear Leukocyte" (PMN) cell-type signature. While similar trends of reduced infiltrate were found for "Inflammatory Monocyte" $\left(\mathrm{GR} 1^{+} / \mathrm{F} 4 / 80^{+}\right.$; WT PBS: $700 \pm$ 209, LPS: $1439 \pm 626$ vs. KO PBS: $603 \pm 187$ LPS: $963 \pm 275)$ and "Macrophage" (GR1-F4/80+' WT PBS: $214 \pm 31$, LPS: $402 \pm 167$ vs. KO PBS: $287 \pm 87$ LPS: $331 \pm 76$ ) monocytic/macrophage-like populations, these results did not reach statistical significance. Together, our initial 3-color flow cytometry data suggested that the primary cell population affected by NR-Traf3 depletion was PMN cells.

To validate our findings, we subsequently performed 7-color flow cytometry on LPS $^{\text {ivt }}$-treated NR-Traf3 WT and KO retinas. In agreement with our initial results, we observed a considerable reduction in LPS-stimulated total myeloid infiltrate (Figure 6, population $\mathrm{A}, \mathrm{CD}_{4} 5^{+} \mathrm{CD} 11 \mathrm{~b}^{+}$) in NR-Traf3 $\mathrm{KO}$ retinas (45\% response compared to WT controls), where the majority of cells affected displayed a granulocytic/PMN signature (Figure 6, population $\mathrm{D}, \mathrm{CD} 45^{+} \mathrm{CD} 11 \mathrm{~b}^{+} \mathrm{F} 4 / 80 \mathrm{low} \mathrm{Ly} 6 \mathrm{G}^{+} \mathrm{Ly} 6 \mathrm{C}^{+}$). Using the more rigorous 7 -color analysis, we also found significant genotype-dependent differences in LPS-stimulated monocyte/macrophage populations (Figure 6, population $\mathrm{B}, \mathrm{CD} 45^{+} \mathrm{CD} 11 \mathrm{~b}^{+} \mathrm{F} 4 / 80^{+} \mathrm{CD} 115^{+} \mathrm{CCR} 2^{+}$ $\mathrm{Ly}_{6 \mathrm{C}}{ }^{+}$; and population $\left.\mathrm{C}, \mathrm{CD}_{4} 5^{+} \mathrm{CD} 11 \mathrm{~b}^{+} \mathrm{F} 4 / 80^{+} \mathrm{CD} 115^{+} \mathrm{CCR} 2^{-} \mathrm{Ly} 6 \mathrm{C}^{+}\right)$, although total cell numbers for these populations were much lower than for the granulocyte population (Figure 6, population D). Thus, our findings support that NR-Traf3 promotes retinal immune cell infiltration following $24 \mathrm{~h}$ LPSivt immune activation. 
(a)

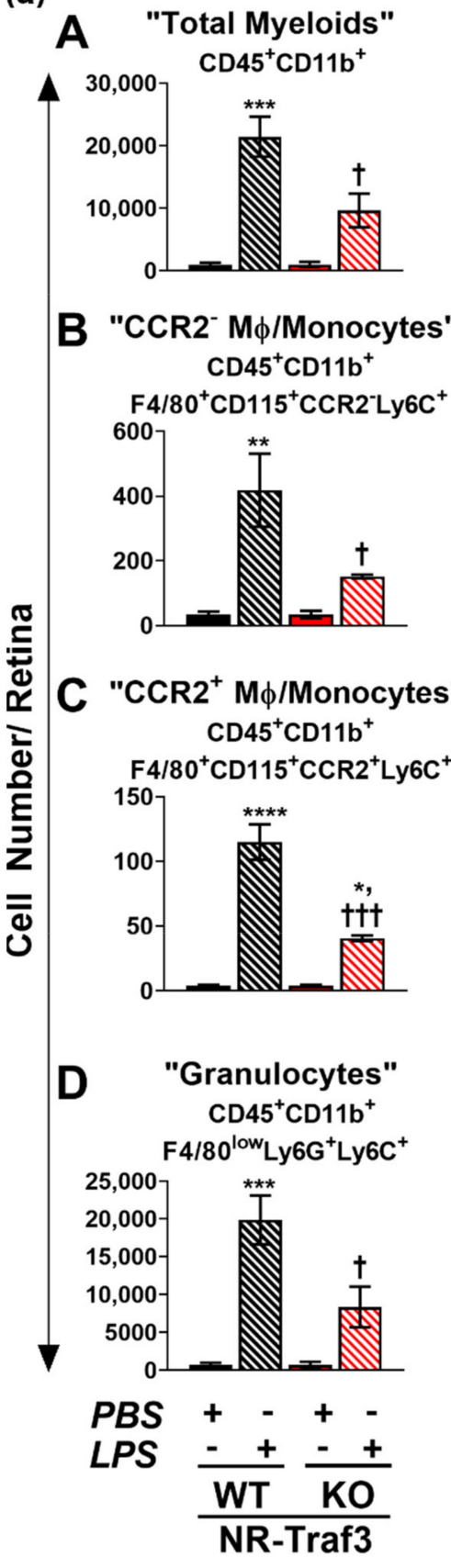

(b) NR-Traf3 WT
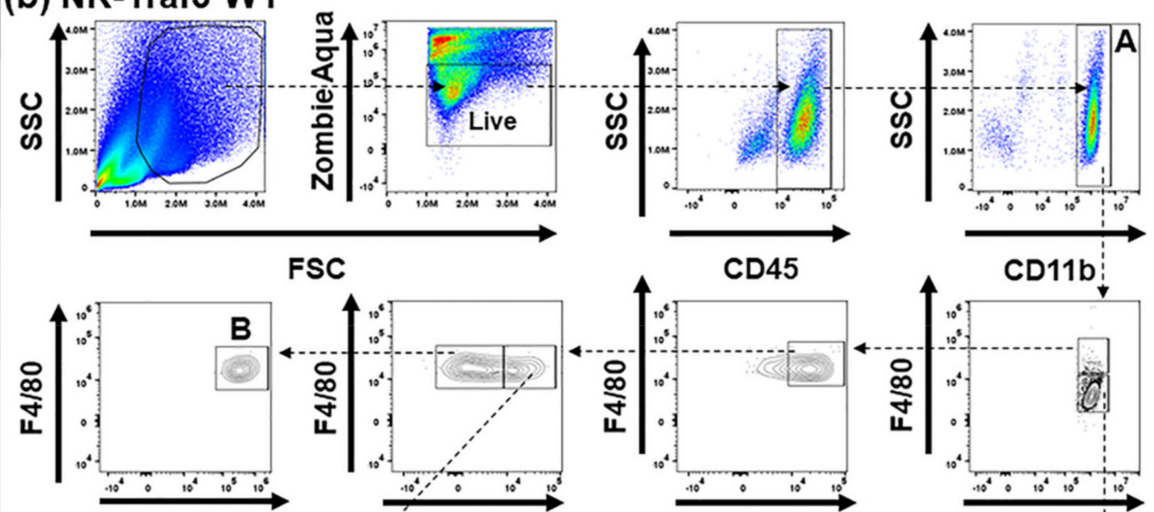

FSC

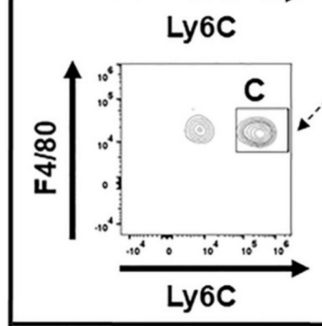

CCR2
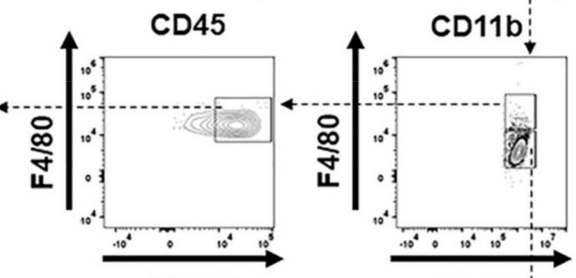

(c) NR-Traf3 KO
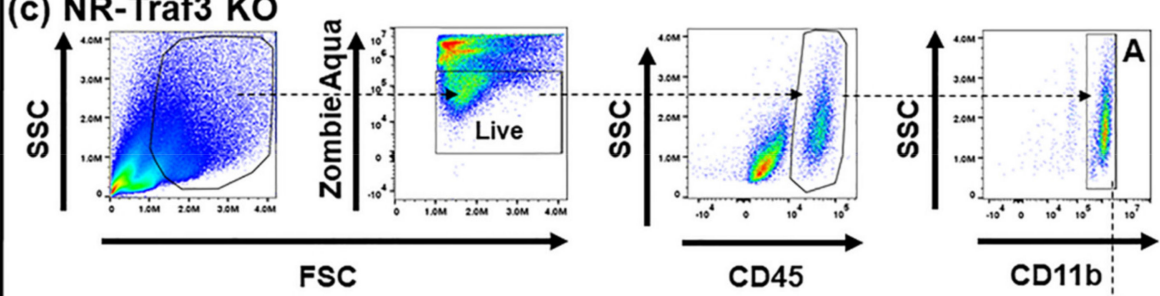

A: Total Myeloids

B: CCR2- M $\$$ / Monocytes

C: CCR2 ${ }^{+}$M $\phi$ / Monocytes

D: Granulocytes

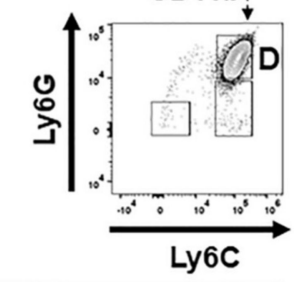

Figure 6. NR-Traf3 promotes LPS-induced retinal immune cell infiltration (7-color). Flow cytometry data representing (a) grouped histogram quantification and (b) representative NR-Traf3 WT and (c) KO dot plots for 7-color flow cytometry detection of immune cells from whole retinal single cell suspensions following $24 \mathrm{~h}$ intravitreal LPS administration. Data for (a) are the mean \pm SEM and were analyzed via two-way ANOVA with Tukey's multiple comparisons post hoc test (LPS effect: ${ }^{* * * *} p<0.0001$, ${ }^{* * *} p<0.001$, ${ }^{* *} p<0.01,{ }^{*} p<0.05$; Genotype effect: ${ }^{++t} p<0.001$, $\left.{ }^{+} p<0.05 ; n=3\right)$. Immune cell populations of interest are designated A-D: A, total myeloids $\left(\mathrm{CD} 45^{+} \mathrm{CD} 11 \mathrm{~b}^{+}\right)$; B, CCR2macrophages (M $\Phi) /$ monocytes $\left(\mathrm{CD} 45^{+} \mathrm{CD} 11 \mathrm{~b}^{+} \mathrm{F} 4 / 80^{+} \mathrm{CD} 115^{+} \mathrm{CCR} 2^{+} \mathrm{Ly} 6 \mathrm{C}^{+}\right)$; $\mathrm{C}, \mathrm{CCR} 2^{+}$macrophages $(\mathrm{M} \Phi) /$ monocytes $\left(\mathrm{CD} 45^{+} \mathrm{CD} 11 \mathrm{~b}^{+} \mathrm{F} 4 / 80^{+} \mathrm{CD} 115^{+} \mathrm{CCR} 2{ }^{-} \mathrm{Ly}_{6 \mathrm{C}^{+}}\right)$; D, granulocytes $\left(\mathrm{CD} 45^{+} \mathrm{CD} 11 \mathrm{~b}^{+} \mathrm{F} 4 / 80^{\mathrm{low}} \mathrm{Ly}_{6 \mathrm{G}} \mathrm{Ly}^{+} \mathrm{C}^{+}\right)$.

\section{Discussion}

Inflammatory diseases of the CNS are not well understood and are further confounded by the unique immune-privileged environment displayed by neuronal tissues. Thus, for the CNS in particular, molecular targeting of novel inflammatory regulators 
with neuronal-specific functions is an attractive strategy for treatment of inflammationassociated neurodegenerative diseases. Due to the greater accessibility of retinal tissue to laboratory manipulations and in vivo assessments, retinal mouse models are frequently used to study retinal inflammatory disease and are considered a precursory conduit for understanding neuronal inflammatory conditions of the brain. It is well known that microglia, astrocytes and the retinal vasculature play important roles in retinal immune response regulation [12,40,41]. Previously, we found that the NR compartment also plays an important role in retinal immunity [13]. The NR houses all retinal neurons as well as Müller glial cells, and accounts for approximately $98.9 \%$ of total cells that comprise retinal tissue in healthy adult mice (Figure 1b) [42]. In our previous study, we found that targeted NR depletion of the Caveolin-1 (Cav1) gene in the NR was sufficient to blunt LPS-induced retinal immune cell infiltration, suggesting that the NR compartment, specifically, plays an important role in retinal immune regulation [13]. Interestingly, we also observed upregulation of the immune modulator TRAF3 in retinal tissue membrane fractions from NR-Cav1 mice. As the role of TRAF3 in the retina has not been investigated, we have generated a NR-Traf3 depletion model to enable examination of retina-specific TRAF3 functions.

At present, there have been no studies conducted on retinal-Traf 3 function. However, gene and protein databases have reported high levels of TRAF3 expression in the retina [27]. Here, we have confirmed that TRAF3 protein is highly expressed in healthy murine retinal tissue, and that the Chx10-Cre model specifically targets retinal, but not brain, neuronal tissue TRAF3 (Figure 2). As the NR contains numerous neuronal subpopulations (as well as Müller glia), we attempted to identify retinal cell type-specific TRAF3 expression via immunofluorescence using several antibody/tissue preparation conditions. However, of all TRAF3 antibodies tested that produced an immunofluorescence signal, none were able to demonstrate a genotype-dependent reduction in immunofluorescence staining that would provide confident identification of neuronal subtype-specific TRAF3 expression. Interestingly, although our Western blot data showed a significant NR-Traf3 KO-dependent reduction in relative TRAF3 protein, we noted that two sets of high-molecular weight non-specific bands were also detected with this antibody (Figure S1a). Thus, while this reagent was able to clearly demonstrate depletion of TRAF3 in our whole retinal lysate samples, future users should use caution and ensure sufficient gel separation of TRAF3 protein from other proteins near the expected molecular weight of $65 \mathrm{kDa}$ that interact with this antibody. While the difficulty in TRAF3 detection via tissue immunofluorescence may be in part due to non-specific binding of this reagent, we also tested various additional antibodies in conjunction with varied antigen-retrieval techniques. We were unable to confidently and specifically identify TRAF3 protein retinas using immunofluorescence, which may require further modification of tissue fixation and antigen-retrieval procedures and/or development of reagents with greater specificity toward retinal TRAF3 protein. Nevertheless, our data support that the NR-Traf3 KO model specifically targets retinal tissue and that the majority of retinal TRAF3 protein expression resides within the NR compartment (Figure 2). This agrees with previous studies that reported antibody detection of TRAF3 in rat spinal cord neurons, mouse brain neurons, and human cerebral and Purkinje neurons, but not astrocytes or oligodendroglia [43,44]. Interestingly, Krajewski, et al. also reported that the level of TRAF3 expression varied among different neuronal subpopulations of the brain [44]. Thus, it is likely that the high NR TRAF3 expression observed in our study is largely due to neuronal-derived expression; however, the level of expression may vary among the various retinal neuronal subtypes and Müller glial-derived TRAF3 expression cannot be excluded. Future studies aim to elucidate retinal cell-specific TRAF3 protein detection via further development of immunofluorescence methods, as well as using alternative flow cytometry and cell culture techniques.

Numerous non-retinal TRAF3 studies have demonstrated a breadth of Traf3 functions, including modulation of cell survival, metabolic, and immune processes [15,18,22,45,46]. Given the complex cellular composition and high metabolic demands of retinal tissue, we first characterized the impact of NR-Traf3 depletion on retinal structure and function 
to determine whether our new NR-Traf3 model was suitable for further manipulations that would allow for reliable assessments of TRAF3 function. Using our in-depth in vivo SD-OCT and histological analyses, we found no apparent overall genotype-dependent defects on retinal tissue architecture, lamination, or patterning (Figure 3 and Figure S2). Interestingly, we did identify differences in the inner nasal zone INL of NR-Traf3 heterozygous animals, which statistically affected the TRT for this retinal zone in HET mice. However, all data considered, we believe this singular difference was likely due to marginal biological variability rather than an indicator of meaningful physiological variances as no statistical differences were found for the INL of other retinal zones, or when comparing thicknesses of any of the other sublayers. Moreover, we observed no differences in tissue morphology by H\&E staining (Table S1). Furthermore, we found that NR-Traf3 depletion did not significantly alter retinal electrophysiology or spatial vision (Figures 4 and 5). Taken together, our data suggest that Traf3 deletion in the NR compartment does not result in developmental or degenerative abnormalities and that the NR-Traf3 mouse is a suitable model for investigations of NR-derived TRAF3 function.

TRAF3 is ubiquitously expressed and global-Traf3 deletion results in neonatal lethality in mice, which is likely due to systemic effects on the immune system that result in a hyperinflammatory state $[19,44]$. In fact, the majority of TRAF3 studies are focused on TRAF3-regulated immune cell-specific functions. Collectively, these studies identified TRAF3 as a cytosolic and nuclear adaptor protein that regulates numerous cell surface receptors and downstream effectors in a highly context- and cell-specific manner in order to regulate various cellular processes involved in immune cell development (natural killer T cells), survival (B cells), and metabolism (myeloid and B cells) [17,18,47]. Additionally, TRAF3 can either positively or negatively modulate inflammatory and anti-viral signaling pathways, which is cell type- and receptor-dependent [47-50]. For an extensive overview on the complexities of Traf 3 functions in immune cells, the reader is referred to previously published reviews $[18,45,47]$.

In recent years, numerous studies have utilized conditional Traf3 knockout models to reveal additional roles for non-immune cell, tissue-specific TRAF3 functions. For example, TRAF3 is expressed in both osteoclasts and osteoblasts, and thus plays important roles in both bone resorption and formation during skeletal development and bone remodeling, respectively [15,51-53]. Here, our data show that NR-Traf3 promotes the retinal immune response by facilitating LPS-induced inflammatory immune cell infiltration (Figure 6 and Figure S3). This agrees with previous data showing that conditional-Traf3 depletion in the liver results in reduced immune infiltration following ischemia/reperfusion injury, whereas hepatic Traf3 overexpression exacerbates the level of infiltrate [54]. In this study, canonical $\mathrm{NFKB}$ signaling pathway effectors and inflammatory cytokine gene expression targets were shown to be similarly upregulated by liver-derived TRAF3 and were assumed to be responsible for the effects on hepatic tissue inflammatory infiltrate. Importantly, another study using conditional depletion of Traf3 in brain-specific neurons showed that TRAF3 also activates canonical inflammatory NFKB signaling in the brain [24]. Thus, it is probable that a primary function of retinal TRAF3 is to regulate retinal immune responses. However, it is conceivable that NR-Traf3 harbors the ability to modulate additional retinal signaling pathways and cellular processes as TRAF3 has been shown to directly and indirectly interact with multiple immune receptors, respond to a variety of upstream stimuli, and regulate molecular effectors belonging to diverse signaling pathways. In the heart, TRAF3 mediates GPCR (G-coupled protein receptor)-stimulated cardiac hypertrophy through activation of TBK1-AKT-mTOR signaling, which results in increased protein synthesis that contributes to the hypertrophic response [23]. In the liver, TRAF3 contributes to hepatic cell death following ischemia/reperfusion injury and to hepatic steatosis following HFD (high-fat diet)-induced diabetes, in part, through promotion of via TAK1-MKK-JNK signaling $[21,54]$. Likewise, in the brain, TRAF3 promotes neuronal cell death partly through TAK1-MKK-JNK activation in both ischemia/reperfusion and subarachnoid hemorrhage injury models $[24,28]$. Zhang et al. also showed that TRAF3 promoted neuronal apoptosis 
following spinal cord injury via thoracic vertebral contusion; however, whether this was also dependent on TAK1-MKK-JNK activation was not investigated in this study [29]. Here, we show that NR-TRAF3 regulates TLR4-specific immune activation by LPS endotoxin administration. Thus, as our previous work in NR-Cav1 KO animals also showed blunted immune cell infiltration, the simultaneous TRAF3 upregulation we observed might reflect the retina's attempt to overcome the inability of NR-Cav1 to promote the immune response (rather than suggest that TRAF3 is responsible for the blunted response) [13]. Our future studies include identification of NR-TRAF3-dependent signaling pathways involved in the retinal immune response to various inflammatory stimuli, as well as developing a more thorough investigation of TRAF3-dependent infiltrating populations, including immune cells of both lymphoid and myeloid lineages. Additionally, we plan to investigate corresponding retinal cytokine/chemokine responses to various inflammatory stimuli, as well as potential interactions between CAV1 and TRAF3. Our novel NR-Traf3 model can also be used to investigate potential non-immune retinal TRAF3 functions.

\section{Conclusions}

To our knowledge, we have generated the first retinal-specific conditional Traf3 knockout mouse model (NR-Traf3 KO) using Chx10-Cre-mediated targeting of the neural retinal (NR) compartment. Our analyses of retinal tissue from NR-Traf3 KO animals suggest that this model provides a valuable tool for investigating neuronal Traf3 functions. Here, we also provide preliminary evidence that NR-Traf3 regulates the retinal immune response as NR-Traf3 ablation was sufficient to blunt LPS-mediated retinal immune cell infiltration. While the precise mechanism by which NR-Traf3 promotes immune infiltration needs further investigation, our study agrees with existing literature that suggests therapeutic targeting of neuronal Traf3 may provide neuroprotection and prevent chronic inflammation that occurs with neurodegenerative diseases of the CNS.

Supplementary Materials: The following are available online at https:/ /www.mdpi.com/article/10.339 0/cells10082068/s1, Figure S1: Effect of NR-Traf3 depletion on retinal TRAF3 protein expression, body weight, brain tissue weight, and average retinal tissue weight. Figure S2: Normal Müller glial and vascular stratification occurs in mouse retinal tissue with NR-Traf3 depletion. Figure S3: NR-Traf3 promotes LPS-induced retinal immune cell infiltration (3-color). Table S1: Detailed SD-OCT analysis for NR-Traf3 adult mouse retinal structure and lamination.

Author Contributions: Conceptualization, J.M.G. and M.H.E.; methodology, J.M.G., G.B.G., G.A.B., D.J.J.C. and M.H.E.; formal analysis, J.M.G., G.B.G., E.A.H. and M.H.E.; investigation, J.M.G., G.B.G. and E.A.H., M.H.E.; resources, G.A.B.; data curation, J.M.G., G.B.G. and M.H.E.; writing-original draft preparation, J.M.G.; writing—review and editing, J.M.G., G.B.G., G.A.B., D.JJ.C. and M.H.E.; visualization, J.M.G. and M.H.E.; supervision, J.M.G., D.J.J.C. and M.H.E.; project administration, J.M.G. and M.H.E.; funding acquisition, J.M.G., G.A.B., D.J.J.C. and M.H.E. All authors have read and agreed to the published version of the manuscript.

Funding: This research was funded by grants from R01EY019494 to M.H.E.; T32EY023202; P30EY021725; an unrestricted grant from Research to Prevent Blindness to the Department of Ophthalmology at the Dean McGee Eye Institute (OUHSC); R01AI28847 to G.A.B.; R01AI053108 to D.J.J.C.; J.M.G. is the recipient of the Postdoctoral OCAST Fellowship grant HF18-008.

Institutional Review Board Statement: All animal studies complied with the Association for Research in Vision and Ophthalmology (ARVO) Statement for the Use of Animals in Ophthalmic and Vision Research and the National Institutes of Health Guide for the Care and Use of Laboratory Animals and were approved by the Institutional Animal Care and Use Committee of the University of Oklahoma Health Sciences Center (protocol number 18-071, approved 16 July 2019).

Informed Consent Statement: Not applicable.

Data Availability Statement: The data supporting the findings of this study are available from the corresponding author upon reasonable request. 
Acknowledgments: We thank Linda Boone in the NEI P30-supported Cellular Imaging Core for expert preparation of tissue sections for immunohistochemistry.

Conflicts of Interest: The authors declare no conflict of interest.

\section{References}

1. Ksander, B.R.; Streilein, J.W. Regulation of the immune response within privileged sites. Chem. Immunol. 1994, 58, 117-145.

2. London, A.; Benhar, I.; Schwartz, M. The retina as a window to the brain-from eye research to CNS disorders. Nat. Rev. Neurol. 2013, 9, 44-53. [CrossRef] [PubMed]

3. Bermel, R.A.; Inglese, M. Neurodegeneration and inflammation in MS: The eye teaches us about the storm. Neurology 2013, 80, 19-20. [CrossRef]

4. Grossniklaus, H.E.; Geisert, E.E.; Nickerson, J.M. Introduction to the Retina. Prog. Mol. Biol. Transl. Sci. 2015, 134, 383-396.

5. Ridge, K.D.; Abdulaev, N.G.; Sousa, M.; Palczewski, K. Phototransduction: Crystal clear. Trends Biochem. Sci. 2003, $28,479-487$. [CrossRef]

6. Neves, G.; Lagnado, L. The retina. Curr. Biol. 1999, 9, R674-R677. [CrossRef]

7. Nickells, R.W.; Howell, G.R.; Soto, I.; John, S.W. Under pressure: Cellular and molecular responses during glaucoma, a common neurodegeneration with axonopathy. Annu. Rev. Neurosci. 2012, 35, 153-179. [CrossRef]

8. Rubsam, A.; Parikh, S.; Fort, P.E. Role of Inflammation in Diabetic Retinopathy. Int. J. Mol. Sci. 2018, 19, 942. [CrossRef]

9. Silverman, S.M.; Wong, W.T. Microglia in the Retina: Roles in Development, Maturity, and Disease. Annu. Rev. Vis. Sci. 2018, 4, 45-77. [CrossRef]

10. Guttenplan, K.A.; Stafford, B.K.; El-Danaf, R.N.; Adler, D.I.; Munch, A.E.; Weigel, M.K.; Huberman, A.D.; Liddelow, S.A. Neurotoxic Reactive Astrocytes Drive Neuronal Death after Retinal Injury. Cell Rep. 2020, 31, 107776. [CrossRef]

11. Detrick, B.; Hooks, J.J. Immune regulation in the retina. Immunol. Res. 2010, 47, 153-161. [CrossRef]

12. Crane, I.J.; Liversidge, J. Mechanisms of leukocyte migration across the blood-retina barrier. Semin. Immunopathol. 2008, 30, 165-177. [CrossRef]

13. Gurley, J.M.; Gmyrek, G.B.; McClellan, M.E.; Hargis, E.A.; Hauck, S.M.; Dozmorov, M.G.; Wren, J.D.; Carr, D.J.J.; Elliott, M.H. Neuroretinal-Derived Caveolin-1 Promotes Endotoxin-Induced Inflammation in the Murine Retina. Investig. Ophthalmol. Vis. Sci. 2020, 61, 19. [CrossRef] [PubMed]

14. Yang, X.D.; Sun, S.C. Targeting signaling factors for degradation, an emerging mechanism for TRAF functions. Immunol. Rev. 2015, 266, 56-71. [CrossRef]

15. Boyce, B.F.; Li, J.; Xing, L.; Yao, Z. Bone Remodeling and the Role of TRAF3 in Osteoclastic Bone Resorption. Front. Immunol. 2018, 9, 2263. [CrossRef]

16. Wallis, A.M.; Bishop, G.A. TRAF3 regulation of inhibitory signaling pathways in B and T lymphocytes by kinase and phosphatase localization. J. Leukoc. Biol. 2018, 103, 1089-1098. [CrossRef]

17. Bishop, G.A.; Stunz, L.L.; Hostager, B.S. TRAF3 as a Multifaceted Regulator of B Lymphocyte Survival and Activation. Front. Immunol. 2018, 9, 2161. [CrossRef]

18. Yi, Z.; Wallis, A.M.; Bishop, G.A. Roles of TRAF3 in T cells: Many surprises. Cell Cycle 2015, 14, 1156-1163. [CrossRef]

19. Xu, Y.; Cheng, G.; Baltimore, D. Targeted disruption of TRAF3 leads to postnatal lethality and defective T-dependent immune responses. Immunity 1996, 5, 407-415. [CrossRef]

20. Xie, P.; Stunz, L.L.; Larison, K.D.; Yang, B.; Bishop, G.A. Tumor necrosis factor receptor-associated factor 3 is a critical regulator of B cell homeostasis in secondary lymphoid organs. Immunity 2007, 27, 253-267. [CrossRef]

21. Wang, P.X.; Zhang, X.J.; Luo, P.; Jiang, X.; Zhang, P.; Guo, J.; Zhao, G.N.; Zhu, X.; Zhang, Y.; Yang, S.; et al. Hepatocyte TRAF3 promotes liver steatosis and systemic insulin resistance through targeting TAK1-dependent signalling. Nat. Commun. 2016, 7, 10592. [CrossRef]

22. Mambetsariev, N.; Lin, W.W.; Wallis, A.M.; Stunz, L.L.; Bishop, G.A. TRAF3 deficiency promotes metabolic reprogramming in B cells. Sci. Rep. 2016, 6, 35349. [CrossRef]

23. Jiang, X.; Deng, K.Q.; Luo, Y.; Jiang, D.S.; Gao, L.; Zhang, X.F.; Zhang, P.; Zhao, G.N.; Zhu, X.; Li, H. Tumor necrosis factor receptor-associated factor 3 is a positive regulator of pathological cardiac hypertrophy. Hypertension 2015, 66, 356-367. [CrossRef]

24. Gong, J.; Li, Z.Z.; Guo, S.; Zhang, X.J.; Zhang, P.; Zhao, G.N.; Gao, L.; Zhang, Y.; Zheng, A.; Zhang, X.F.; et al. NeuronSpecific Tumor Necrosis Factor Receptor-Associated Factor 3 Is a Central Regulator of Neuronal Death in Acute Ischemic Stroke. Hypertension 2015, 66, 604-616. [CrossRef] [PubMed]

25. He, J.Q.; Saha, S.K.; Kang, J.R.; Zarnegar, B.; Cheng, G. Specificity of TRAF3 in its negative regulation of the noncanonical NF-kappa B pathway. J. Biol. Chem. 2007, 282, 3688-3694. [CrossRef] [PubMed]

26. Jia, R.; Ma, J.; Xiang, S.; Meng, W.; Wang, N. Caerulin-induced pro-inflammatory response in macrophages requires TRAF3-p38 signaling activation. Biochem. Biophys. Res. Commun. 2017, 494, 358-364. [CrossRef]

27. Stelzer, G.; Rosen, N.; Plaschkes, I.; Zimmerman, S.; Twik, M.; Fishilevich, S.; Stein, T.I.; Nudel, R.; Lieder, I.; Mazor, Y.; et al. The GeneCards Suite: From Gene Data Mining to Disease Genome Sequence Analyses. Curr. Protoc. Bioinform. 2016, 54, 1-30. [CrossRef] 
28. Zhou, Y.; Tao, T.; Liu, G.; Gao, X.; Gao, Y.; Zhuang, Z.; Lu, Y.; Wang, H.; Li, W.; Wu, L.; et al. TRAF3 mediates neuronal apoptosis in early brain injury following subarachnoid hemorrhage via targeting TAK1-dependent MAPKs and NF-kappaB pathways. Cell Death Dis. 2021, 12, 10. [CrossRef]

29. Zhang, Z.; Shen, L.; Yan, Y. MiR-139-5p alleviates neural cell apoptosis induced by spinal cord injury through targeting TRAF3. Acta Biochim. Pol. 2020, 67, 359-365. [CrossRef]

30. Rowan, S.; Cepko, C.L. Genetic analysis of the homeodomain transcription factor Chx10 in the retina using a novel multifunctional BAC transgenic mouse reporter. Dev. Biol. 2004, 271, 388-402. [CrossRef] [PubMed]

31. Reagan, A.; Gu, X.; Hauck, S.M.; Ash, J.D.; Cao, G.; Thompson, T.C.; Elliott, M.H. Retinal Caveolin-1 Modulates Neuroprotective Signaling. Adv. Exp. Med. Biol. 2016, 854, 411-418. [CrossRef]

32. Prusky, G.T.; Alam, N.M.; Beekman, S.; Douglas, R.M. Rapid quantification of adult and developing mouse spatial vision using a virtual optomotor system. Investig. Ophthalmol. Vis. Sci. 2004, 45, 4611-4616. [CrossRef]

33. Sethna, S.; Chamakkala, T.; Gu, X.; Thompson, T.C.; Cao, G.; Elliott, M.H.; Finnemann, S.C. Regulation of Phagolysosomal Digestion by Caveolin-1 of the Retinal Pigment Epithelium Is Essential for Vision. J. Biol. Chem. 2016, 291, 6494-6506. [CrossRef]

34. Mandal, M.N.; Moiseyev, G.P.; Elliott, M.H.; Kasus-Jacobi, A.; Li, X.; Chen, H.; Zheng, L.; Nikolaeva, O.; Floyd, R.A.; Ma, J.X.; et al. Alpha-phenyl-N-tert-butylnitrone (PBN) prevents light-induced degeneration of the retina by inhibiting RPE65 protein isomerohydrolase activity. J. Biol. Chem. 2011, 286, 32491-32501. [CrossRef]

35. Winkler, B.S. The electroretinogram of the isolated rat retina. Vis. Res. 1972, 12, 1183-1198. [CrossRef]

36. Anderson, R.E.; Maude, M.B.; McClellan, M.; Matthes, M.T.; Yasumura, D.; LaVail, M.M. Low docosahexaenoic acid levels in rod outer segments of rats with $\mathrm{P} 23 \mathrm{H}$ and S334ter rhodopsin mutations. Mol. Vis. 2002, 8, 351-358. [PubMed]

37. Zhu, S.; Yam, M.; Wang, Y.; Linton, J.D.; Grenell, A.; Hurley, J.B.; Du, J. Impact of euthanasia, dissection and postmortem delay on metabolic profile in mouse retina and RPE/choroid. Exp. Eye Res. 2018, 174, 113-120. [CrossRef]

38. Arora, M.; Poe, S.L.; Oriss, T.B.; Krishnamoorthy, N.; Yarlagadda, M.; Wenzel, S.E.; Billiar, T.R.; Ray, A.; Ray, P. TLR4/MyD88induced CD11b+Gr-1 int F4/80+ non-migratory myeloid cells suppress Th2 effector function in the lung. Mucosal Immunol. 2010, 3, 578-593. [CrossRef]

39. Rosenbaum, J.T.; Woods, A.; Kezic, J.; Planck, S.R.; Rosenzweig, H.L. Contrasting ocular effects of local versus systemic endotoxin. Investig. Ophthalmol. Vis. Sci. 2011, 52, 6472-6477. [CrossRef]

40. Vecino, E.; Rodriguez, F.D.; Ruzafa, N.; Pereiro, X.; Sharma, S.C. Glia-neuron interactions in the mammalian retina. Prog. Retin. Eye Res. 2016, 51, 1-40. [CrossRef] [PubMed]

41. Rathnasamy, G.; Foulds, W.S.; Ling, E.A.; Kaur, C. Retinal microglia-A key player in healthy and diseased retina. Prog. Neurobiol. 2019, 173, 18-40. [CrossRef]

42. Macosko, E.Z.; Basu, A.; Satija, R.; Nemesh, J.; Shekhar, K.; Goldman, M.; Tirosh, I.; Bialas, A.R.; Kamitaki, N.; Martersteck, E.M.; et al. Highly Parallel Genome-wide Expression Profiling of Individual Cells Using Nanoliter Droplets. Cell 2015, 161, 1202-1214. [CrossRef]

43. Wu, Y.; Zheng, M.; Wang, S.; Song, C.; Wang, C.; Xiao, Y.; Xu, L.; Xu, X. Spatiotemporal pattern of TRAF3 expression after rat spinal cord injury. J. Mol. Histol. 2014, 45, 541-553. [CrossRef]

44. Krajewski, S.; Zapata, J.M.; Krajewska, M.; VanArsdale, T.; Shabaik, A.; Gascoyne, R.D.; Reed, J.C. Immunohistochemical analysis of in vivo patterns of TRAF-3 expression, a member of the TNF receptor-associated factor family. J. Immunol. 1997, 159, 5841-5852.

45. Bishop, G.A.; Xie, P. Multiple roles of TRAF3 signaling in lymphocyte function. Immunol. Res. 2007, 39, 22-32. [CrossRef]

46. Chen, Z.; Canet, M.J.; Sheng, L.; Jiang, L.; Xiong, Y.; Yin, L.; Rui, L. Hepatocyte TRAF3 promotes insulin resistance and type 2 diabetes in mice with obesity. Mol. Metab. 2015, 4, 951-960. [CrossRef] [PubMed]

47. Bishop, G.A. TRAF3 as a powerful and multitalented regulator of lymphocyte functions. J. Leukoc. Biol. 2016, 100, 919-926. [CrossRef]

48. Lin, W.W.; Hildebrand, J.M.; Bishop, G.A. A Complex Relationship between TRAF3 and Non-Canonical NF-kappaB2 Activation in B Lymphocytes. Front. Immunol. 2013, 4, 477. [CrossRef] [PubMed]

49. Xie, P.; Poovassery, J.; Stunz, L.L.; Smith, S.M.; Schultz, M.L.; Carlin, L.E.; Bishop, G.A. Enhanced Toll-like receptor (TLR) responses of TNFR-associated factor 3 (TRAF3)-deficient B lymphocytes. J. Leukoc. Biol. 2011, 90, 1149-1157. [CrossRef] [PubMed]

50. Xie, X.; Jin, J.; Zhu, L.; Jie, Z.; Li, Y.; Zhao, B.; Cheng, X.; Li, P.; Sun, S.C. Cell type-specific function of TRAF2 and TRAF3 in regulating type I IFN induction. Cell Biosci. 2019, 9, 5. [CrossRef]

51. Yao, Z.; Lei, W.; Duan, R.; Li, Y.; Luo, L.; Boyce, B.F. RANKL cytokine enhances TNF-induced osteoclastogenesis independently of TNF receptor associated factor (TRAF) 6 by degrading TRAF3 in osteoclast precursors. J. Biol. Chem. 2017, 292, 10169-10179. [CrossRef] [PubMed]

52. Yao, Z.; Xing, L.; Boyce, B.F. NF-kappaB p100 limits TNF-induced bone resorption in mice by a TRAF3-dependent mechanism. J. Clin. Investig. 2009, 119, 3024-3034. [CrossRef] [PubMed]

53. Xiu, Y.; Xu, H.; Zhao, C.; Li, J.; Morita, Y.; Yao, Z.; Xing, L.; Boyce, B.F. Chloroquine reduces osteoclastogenesis in murine osteoporosis by preventing TRAF3 degradation. J. Clin. Investig. 2014, 124, 297-310. [CrossRef]

54. Hu, J.; Zhu, X.H.; Zhang, X.J.; Wang, P.X.; Zhang, R.; Zhang, P.; Zhao, G.N.; Gao, L.; Zhang, X.F.; Tian, S.; et al. Targeting TRAF3 signaling protects against hepatic ischemia/reperfusions injury. J. Hepatol. 2016, 64, 146-159. [CrossRef] 